\title{
Antimicrobial Resistance or Increased Antimicrobial Resistance Rates- which One is Reality?
}

Habip Gedik*

Department of Infectious Diseases and Clinical Microbiology, Ministry of Health Bakırköy Sadi Konuk Training and Research Hospital, Istanbul

Antimicrobial Resistance (AR) is a response of microorganisms by varying mechanisms against antimicrobials to survive and to ensure the continuation of their species. Antimicrobial resistance is a challenge for humanity, but essential survival of microorganisms. Microorganisms become resistant by exchange or receiving resistance genes from other microorganisms or environment. Antimicrobial resistance mechanisms, such as serine and metallo- $\beta$-lactamases, some serine $\beta$-lactamases, were reported to exist four billion years ago $[1,2]$. Therefore, AR will exist in the future as well. The real problem is increased antimicrobial resistance rates through the worldwide, not the existence of antimicrobial resistance. Why have the antimicrobial resistance rates steadily been increasing in the globe? This problem is definitely associated with multifactor. AR is spreading from hospitals to community with colonized patients or people, antibiotic containing sewage, and antibiotic-based drugs used in the growth of animals and plants. Only antimicrobial stewardship cannot solve this problem, just decreases the rates little. Hospitals, long-term care facilities, nursing home facilities where antibiotics are often and excessively used, have a local flora that is being formed by resistant organisms. There are two main steps in development and spreading of AR. The first one is the emergence of resistant microbial flora, and second one is spreading of those bacteria by living and inanimate objects. So, what should be done? Antimicrobial stewardship decreases selection and rates of resistant microorganisms, but cannot eliminate completely. Most of the physicians working in primary care are pressed to prescript an antibiotic by patients or their relatives due to upper respiratory tract infections that commonly occur by virus, and bacteriuria that is generally colonization secondary to invasive intervention and not needed an antibiotic therapy [3]. Physicians do not feel comfortable their selves for fear of malpractice, lawsuits, and inadequate treatment. The founders and organizers of the health care systems should make an antimicrobial stewardship program to support the physicians for less antibiotic prescriptions and making them feel safe with guidelines and law enforcement. Infection control measures should be performed strictly, and cohorting should be made for people colonized with resistant microorganisms at hospitals, long-term care facilities, nursing home facilities where antibiotics are often and excessively used antimicrobials.

Cleaning staff should be educated about resistant microorganisms, healthcare associated infections, appropriate cleaning of patient rooms, glove changing at each patient room. Those are important to prevent spreading of microorganisms in the settings. It was reported that 70 and $76 \%$ of environmental sites were unacceptable after cleaning [4]. Cleaning should be monitored by microbiological culture or ATP bioluminescence. Cleaning staff should be trained about the cleansing and the importance of appropriate and exact cleansing as one measure of infection. They also should be monitored about whether they officiate appropriately. Recruiting of people with obsessive-compulsive disorder in terms of cleanliness at cleansing of very dirty places, and nursing houses and critical sections as psychotherapy would be helpful as a utopian idea. They are very obsessive to germs and contamination. However, healthcare staffs, especially doctors are not responsive to hand hygiene. Hands of healthcare staff take a role in transmission of bacteria to patients. Even if health workers are followed-up for hand hygiene, one hundred percent of compliance could not be provided. There are many barriers to achieve compliance. In particular, employees feel free their selves for hand hygiene in outside of the working hours with the reduction of supervising of employees. All healthcare workers, especially doctors should be motivated for hand hygiene, and be informed about transmission of bacteria that cause death of patient and turn waste their work and effort by their hands. Antibiotics are used not only in hospitals, but also particularly in animal breeding. Although their use is limited in many countries, there are some countries or regions where it cannot be achieved. This problem allows colonization of resistant bacteria in animals and the soil. Antibiotic or antibiotic-based drugs should be forbidden not only at hospitals, but also in animal husbandry and agriculture. Hospital wastewaters are containing antibiotics, and also increase resistant bacteria colonization in the environment and soil. Consequently, the development and spreading of AR depend on many factors. Only restriction of antibiotic use does not solve this problem. It is obvious that much more projects and interventions that are performed in cooperation of all determinants including health workers, hospital administrators, national health institutes, international health policy determinants, the agriculture and livestock sectors, the pharmaceutical industry are needed. Illustrious persons, including actors, actresses, sportsmen, etc. should also be involved in awareness of this problem. Movies and TV series may benefit in the description of this problem through the worldwide.

\section{References}

1. Hall BG, Barlow M (2004) Evolution of the serine beta-lactamases: past, present and future. Drug Resist Updat 7: 111-123.

2. Garau G, Di Guilmi AM, Hall BG (2005) Structure-based phylogeny of the metallo-beta-lactamases. Antimicrob Agents Chemother 49: 2778-2784.

3. McDonagh M, Peterson K, Winthrop K, Cantor A, Holzhammer B, et al. (2016) Improving antibiotic prescribing for uncomplicated acute respiratory tract infections. Comparative Effectiveness Reviews.

4. Griffith CJ, Cooper RA, Gilmore J, Davies C, Lewis (2000) An evaluation of hospital cleaning regimes and standards. J Hosp Infect 45: 19-28.

*Corresponding author: Habip Gedik, Department of Infectious Diseases and Clinical Microbiology, Ministry of Health Bakırköy Sadi Konuk Training and Research Hospital, İstanbul, Tel: +902124147200; E-mail: habipgedik@yahoo. com

Received: September 13, 2016; Accepted: September 19, 2016; Published: September 26, 2016

Citation: Gedik H (2016) Antimicrobial Resistance or Increased Antimicrobia Resistance Rates- which One is Reality? J Infect Dis Med 1: 103. doi: 10.4172/25761420.1000101

Copyright: (C) 2016 Gedik $\mathrm{H}$. This is an open-access article distributed under the terms of the Creative Commons Attribution License, which permits unrestricted use, distribution, and reproduction in any medium, provided the original author and source are credited. 